\title{
Ligamento periodontal y pulpa radicular en la estimación de la edad cronológica en una población peruana
}

\section{Periodontal ligament and root pulp in the estimation of chronological age in Peruvian population}

\begin{abstract}
Resumen
Objetivo. Determinar la eficacia entre los métodos radiográficos del ligamento periodontal y pulpa radicular en el tercer molar inferior para la estimación de la edad cronológica. Métodos. Diseño transversal, se trabajó con una muestra de 347 imágenes radiográficas panorámicas digitales (187 hombres, 160 mujeres) comprendidos entre los 16 a 74 ańos, La estimación de la edad cronológica se basó en la clasificación de estadios propuestos por Olze, con una correlación intraexaminador de $(0,870 ; 0,902)$ e inter-examinador $(0,907 ; 0,920)$ por la Prueba de Kappa de Cohen. La relación entre la edad cronológica, los estadios del ligamento periodontal y pulpa radicular se determinó a través de pruebas de correlación de Spearman y Pearson, la prueba de Wilcoxon estimó la diferencia entre las piezas dentarias 38 y 48 con un nivel de significancia $p<0,05$. Resultados. La correlación de Spearman fue positiva mediana y alta y la correlación de Pearson positiva moderada y alta. La diferencia fue significativa entre las terceras molares inferiores en ambos métodos. Además, no hubo diferencia significativa entre la edad cronológica y la estimación de la edad con el estudio de la pulpa radicular y con el estudio del ligamento periodontal. Conclusión. El método radiográfico de la pulpa radicular en el tercer molar inferior derecho es más preciso que del ligamento periodontal, así mismo, el método de estudio radiográfico del ligamento periodontal del tercer molar inferior izquierdo es más eficaz que de la pulpa radicular.
\end{abstract}

Palabras clave: Odontología forense; Ligamento periodontal; Pulpa dental; Tercer molar (fuente: DeCS BIREME).

\section{Artículo Original}

Denisse Carhuamaca-León ${ }^{1, a}$, Daniel Suárez-Ponce ${ }^{1, b}$, Teresa Evaristo-Chiyong ${ }^{1,0}$

${ }^{1}$ Universidad Nacional Mayor de San Marcos, Facultad de Odontología. Lima, Perú.

a Cirujano Dentista.

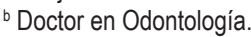

${ }^{c}$ Doctora en Odontología.

\section{Correspondencia:}

Daniel Guillermo Suárez Ponce: dsuarezp@unmsm.edu.pe Facultad de Odontología de la Universidad Nacional Mayor de San Marcos

Calle Germán Amezaga N 375, Facultad de Odontología Ciudad Universitaria, Lima 1, Cercado de Lima, Lima-Perú ORCID: 0000-0003-1287-527X

\section{Coautores:}

Denisse Carhuamaca-León: denisseanny@hotmail.com ORCID: 0000-0002-9952-2903

Teresa A Evaristo-Chiyong: tevaristoc@unmsm.edu.pe ORCID: 0000-0001-7129-5617

\section{Editora:}

Luz Dahiana Alfaro Carballido

Universidad de San Martín de Porres, Lima, Perú

Conflicto de intereses: los autores declaran no tener conflictos de interés.

Fuente de financiamiento: autofinanciado.

Recibido: $16 / 01 / 20$

Aceptado: 04/04/20

Publicado: 09/05/20

\footnotetext{
Abstract

Objective. Determine the effectiveness between radiographic methods of the periodontal ligament and root pulp of the lower third molars, in the estimation of chronological age. Methods. Through a cross-sectional design, using a sample of 347 digital panoramic radiographic images (187 males, 160 females) between 16 and 74 years old. The estimation of chronological age was based on the classification of stages proposed by Olze, with a intra-examiner and inter-examiner agreement was determined by Cohen's Kappa Test. The relationship among the chronological age, the stages of the periodontal ligament
} 
and the root pulp were assessed using Spearman and Pearson rank-order correlation. The Wilcoxon test estimated the difference between dental pieces 38-48, with a level of significance $\mathrm{p}<0.05$. Results. There was a medium and long positive correlation. There was a significant difference between the lower third molars in both methods. Also, there wasn't a significant difference between age chronology and age estimation with the study of root pulp of dental and with the study of periodontal ligament. Conclusions. The radiographic method of the root pulp in the right lower third molar is more precise than the periodontal ligament. Likewise, the radiographic study method of the periodontal ligament of the left lower third molar is more efficient than of the root pulp.

Keywords: Forensic dentistry; Periodontal ligament; Dental pulp; Third molar (source: MeSH NLM).

\section{Introducción}

Se ha demostrado la fiabilidad y utilidad de emplear la dentición humana para la estimación de la edad cronológica mediante la aplicación de métodos morfológicos, bioquímicos y radiológicos ${ }^{1}$.

La evaluación radiográfica dental es una forma fácil y no invasiva de analizar los dientes, en la que se ha desarrollado varios métodos de estadificación de edad basados en el desarrollo del tercer molar inferior y superior ${ }^{2}$. El tercer molar se mineraliza por completo a la edad de los 20 ańos aproximadamente, luego de esto se dificulta estimar la edad ${ }^{3}$.

En 1950, Gustafson proporcionó el primer método científico para la determinación de la edad dental de un individuo ${ }^{4}$. El método de Demirjian y colaboradores propuesta en 1973, establecen un método para estimar la edad basada en ocho estadios de formación de la tercera molar ${ }^{5}$, la literatura señala una fuerte correlación entre los estadios de Demirjian en el tercer molar y la edad cronológica ${ }^{6}$. En 1995, Kvaal y colaboradores desarrollan un método para la estimación de la edad cronológica de un adulto joven basado en el tamaño de la pulpa en radiografías dentales periapicales ${ }^{7}$. Bosmans y colaboradores y Paewinsky y colaboradores aplicaron este método en ortopantomogramas digitales ${ }^{8}$. Kvaal y Solheim presentaron un método en la que las medidas radiológicas (medida del largo de la pulpa, medida del largo radicular en la superficie mesial, ancho de la pulpa en unión cemento esmalte, ancho pulpar en la mitad de la raíz, y el ancho radicular en la mitad de la raíz) y morfológicas (traslucidez apical y retracción del ligamento periodontal en milímetros) están combinadas para estimar la edad de un individuo ${ }^{4}$. En el 2007, Cameriere y colaboradores publicaron un método para la evaluación de la edad cronológica basado en la relación entre la edad y la medición de la relación pulpa y área del diente ${ }^{9}$.

Olze y colaboradores ${ }^{10}$ consideraron dos tipos de métodos para estimar la edad, la visibilidad radiográfica del ligamento periodontal y la pulpa radicular de las terceras molares inferiores con desarrollo completo de la mineralización y raíces con ápices cerrados, clasificados en estadios, indicando que cuando aumenta la edad cronológica aumenta el estadio y son considerados válidos para estimar la edad sobre los 18 o 21 ańos ${ }^{11,12}$.
Estos métodos, permiten relacionar la radiolucidez del ligamento periodontal y la pulpa radicular con la edad cronológica, por lo que es de primordial conocimiento analizar las terceras molares de pacientes jóvenes y adultos, siendo la edad cronológica un dato esencial en la aplicación de las normas jurídicas en el Perú, que refieren que todo ser humano es apto de adquirir derechos y deberes según el margen de edad; siendo la edad relevante a partir de los 18 años, porque se considera mayor de edad capaz de asumir sus obligaciones, o la edad entre 14 y 17 ańos en que se aplica determinadas leyes constitucionales ${ }^{13}$.

El presente trabajo tuvo como objetivo determinar la eficacia entre los métodos radiográficos del ligamento periodontal y pulpa radicular de las terceras molares inferiores, en la estimación de la edad cronológica.

\section{Métodos}

Diseño del estudio. El estudio fue de tipo observacional, transversal. Un total de 347 imágenes radiográficas panorámicas digitales de pacientes, entre los 16 y 74 años fueron analizadas; 160 pertenecían al sexo femenino y 187 al sexo masculino. Las radiografías fueron obtenidas de la base de datos de la clínica de imagenología desde el mes de enero del 2015 hasta octubre del 2017. Se excluyeron aquellas sin nitidez o con imagen de la densidad de tejidos blandos a nivel de tercera molar inferior compatible con inclusión subgingival, imágenes superpuestas que alteren la anatomía radiológica normal de la pulpa radicular y el ligamento periodontal, o con presencia de terceras molares inferiores de un solo lado, en posición impactada o retenida, con formación incompleta de la formación radicular, que se observe imagen radiolúcida (lesión cariosa) e imagen radiopaca (tratamientos restaurativos y/o endodónticos) en la raíz.

Procedimiento de recolección de datos. La estimación de la edad cronológica se basó en la clasificación de estadios propuestos por Olze y colaboradores ${ }^{10-12}$, para el análisis de la visibilidad radiográfica del ligamento periodontal (Figura 1) y de la pulpa radicular de las terceras molares inferiores (Figura 2).

Con la finalidad de establecer un adecuado análisis y evitar el error de método, se realizó la calibración con un especialista de Radiología Bucal y Maxilofacial, para ello se analizaron 84 terceras molares inferiores. 

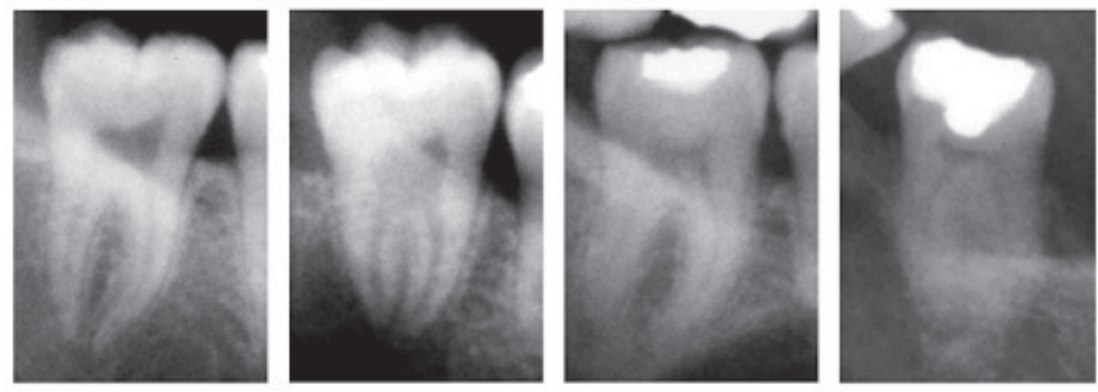

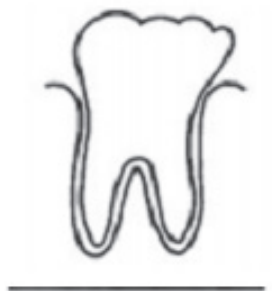

o

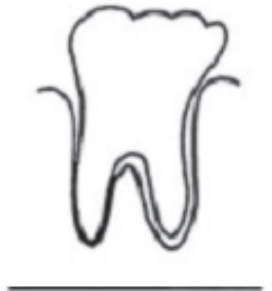

1

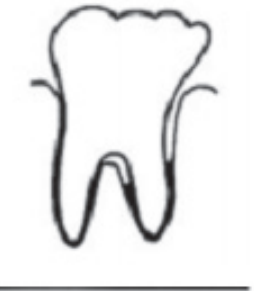

2

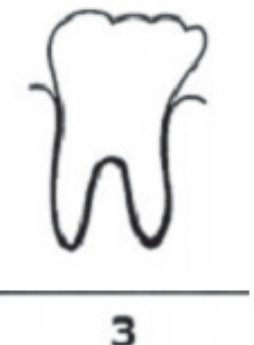

3

Figura 1. Estadios del ligamento periodontal de la raíz de las terceras molares inferiores para estimar la edad Fuente: Olze A y colaboradores, $2010^{10}$

Estadio 0: El ligamento periodontal es visible a lo largo de la longitud total de todas las raíces.

Estadio 1: El ligamento periodontal es invisible en una de las raíces desde el ápice hasta más de la mitad de la raíz.

Estadio 2: El ligamento periodontal es invisible a lo largo de casi la longitud completa de una raíz, o parte de a lo largo de las dos raíces, o ambas.

Estadio 3: El ligamento periodontal es invisible a lo largo de casi la longitud total de dos raíces.

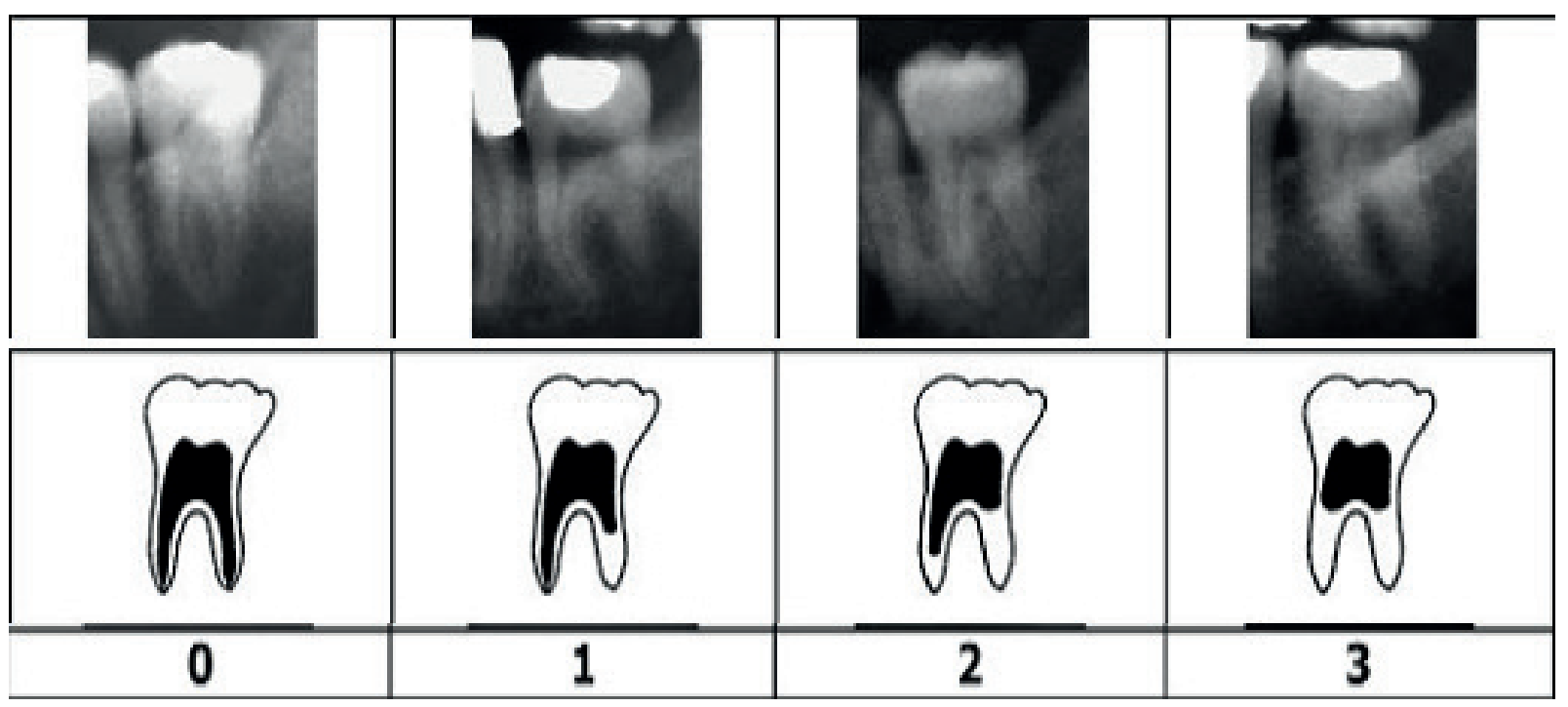

Figura 2. Estadios de la pulpa de la raíz de las terceras molares inferiores para estimar la edad Fuente: Olze A y colaboradores, $2010^{12}$

Estadio 0: El lumen de todos los conductos radiculares es visible todo el recorrido hasta el ápice.

Estadio 1: El lumen de uno de los conductos radiculares no está completamente visible hasta el ápice.

Estadio 2: El lumen de dos conductos radiculares no está completamente visible hasta el ápice, o un conducto debe estar virtualmente invisible en la longitud total, o ambas.

Estadio 3: El lumen de dos conductos radiculares está virtualmente invisible en la longitud total. 
Se acondicionó el ambiente a oscuras, ampliándose la imagen de la tercera molar inferior del lado derecho en un primer momento y luego del lado izquierdo, invirtiendo el contraste de la imagen digital, haciendo uso de las escalas de grises para una mejor visualización radiográfica del ligamento periodontal y de la pulpa radicular. Se aplicó el coeficiente Kappa de Cohen para determinar la confiabilidad interobservador $(0,87 \mathrm{en} \mathrm{el}$ método del ligamento periodontal y de 0,902 en el método de la pulpa radicular) e intraobservador $(0,907$ en el método del ligamento periodontal y de 0,920 en el método de la pulpa radicular). Se registró el número de identificación, sexo, fecha de nacimiento del paciente, fecha de la toma radiográfica, estadio del estudio radiográfico del ligamento periodontal y de la pulpa radicular según el lado de la tercera molar inferior.

Se calculó la edad decimal restando la fecha de la toma radiográfica con la fecha de nacimiento.

Análisis estadístico. El procesamiento de datos se realizó mediante el Software IBM SPSS Statistics v. 23 (IBM, Armonk, New York, USA), para determinar la relación entre los estadios de la edad dental con la edad cronológica; se empleó los coeficientes de correlación de Spearman y Pearson, y para evaluar las diferencias significativas de los estadios del ligamento periodontal y pulpa radicular entre el lado mandibular derecho e izquierdo de la tercera molar inferior se aplicó la prueba de Wilcoxon, siendo el nivel de significancia asumido $(p<0,05)$.

\section{Resultados}

Se determina que el inicio más temprano del estadio 0 del ligamento periodontal es a los 16,57 años (masculino) y 23,90 años (femenino), el estadio 1 a los 17,03 años (masculino) y 17,54 años (femenino) y el estadio 2 a los 24,56 años (masculino) y 26,55 años (femenino) (Tabla 1).

Se establece que el inicio más temprano del estadio 0 de la pulpa radicular es a los 16,57 ańos (masculino) y 17,54 años (femenino), el estadio 1 a los 17,56 ańos (masculino) y 17,86 (femenino) (Tabla 2).

Se observa una correlación positiva entre la edad cronológica y los métodos de estudio en ambos lados del tercer molar, en el estudio radiográfico del ligamento periodontal presenta una fuerza de relación alta en el sexo masculino y mediana en el femenino y en el estudio de la pulpa radicular se observa una fuerza de relación alta en ambos sexos (Tabla 3).

Al comparar la edad cronológica con la estimada mediante el tercer molar derecho se observó que existe una subestimación de la edad al utilizar el método del ligamento periodontal, no encontrándose diferencias estadísticamente significativas con la edad estimada por

Tabla 1. Edad cronológica según los estadios del estudio radiográfico del ligamento periodontal de las terceras molares inferiores

\begin{tabular}{|c|c|c|c|c|c|c|c|c|c|c|c|}
\hline \multirow[t]{2}{*}{ Estadio } & \multirow[t]{2}{*}{ Diente } & \multicolumn{5}{|c|}{ Masculino } & \multicolumn{5}{|c|}{ Femenino } \\
\hline & & Media & $\mathrm{DE}^{*}$ & Mediana & Min & $\operatorname{Max}$ & Media & $\mathrm{DE}^{*}$ & Mediana & Min & $\operatorname{Max}$ \\
\hline \multirow[t]{2}{*}{0} & 38 & 25,33 & 6,51 & 23,36 & 16,57 & 47,47 & 25,96 & 5,94 & 24,31 & 17,65 & 47,53 \\
\hline & 48 & 24,93 & 5,56 & 23,64 & 16,57 & 44,80 & 25,47 & 5,76 & 23,90 & 17,65 & 51,41 \\
\hline \multirow[t]{2}{*}{1} & 38 & 32,53 & 11,25 & 29,40 & 17,93 & 74,16 & 32,04 & 9,95 & 29,21 & 17,54 & 54,79 \\
\hline & 48 & 30,45 & 10,20 & 28,36 & 17,03 & 63,93 & 31,52 & 9,22 & 29,87 & 17,54 & 54,79 \\
\hline \multirow[t]{2}{*}{2} & 38 & 50,3 & 10,64 & 49,97 & 24,56 & 69,02 & 52,12 & 9,60 & 52,03 & 41,13 & 73,54 \\
\hline & 48 & 49,30 & 11,84 & 49,65 & 24,56 & 74,16 & 47,70 & 13,07 & 49,89 & 26,55 & 73,54 \\
\hline
\end{tabular}

$\mathrm{DE}^{*}=$ Desviación estándar

Min=Mínimo, Max=Máximo

Tabla 2. Edad cronológica según los estadios del estudio radiográfico de la pulpa radicular de las terceras molares inferiores

\begin{tabular}{|c|c|c|c|c|c|c|c|c|c|c|c|}
\hline \multirow[b]{2}{*}{ Estadio } & \multirow[b]{2}{*}{ Diente } & \multicolumn{5}{|c|}{ Masculino } & \multicolumn{5}{|c|}{ Femenino } \\
\hline & & Media & $\mathrm{DE}^{*}$ & Mediana & Min & Max & Media & $\mathrm{DE}^{*}$ & Mediana & Min & Max \\
\hline \multirow[t]{2}{*}{0} & 38 & 25,47 & 6,63 & 23,90 & 16,57 & 50,65 & 23,84 & 4,46 & 22,73 & 17,54 & 44,19 \\
\hline & 48 & 23,69 & 4,71 & 22,94 & 16,57 & 35,80 & 24,28 & 4,88 & 23,35 & 17,54 & 44,19 \\
\hline \multirow[t]{2}{*}{1} & 38 & 30,76 & 9,32 & 29,39 & 17,56 & 57,38 & 32,18 & 9,40 & 28,90 & 19,72 & 62,00 \\
\hline & 48 & 31,74 & 10,57 & 29,54 & 17,56 & 69,02 & 30,11 & 9,05 & 28,25 & 17,86 & 59,04 \\
\hline \multirow[t]{2}{*}{2} & 38 & 54,53 & 11,06 & 56,33 & 31,79 & 74,16 & 44,65 & 12,74 & 43,74 & 21,43 & 73,54 \\
\hline & 48 & 48,75 & 12,15 & 49,97 & 24,56 & 74,16 & 47,13 & 11,96 & 44,91 & 23,90 & 73,54 \\
\hline
\end{tabular}

$\mathrm{DE}^{*}=$ Desviación estándar

Min=Mínimo, Max=Máximo 
la pulpa radicular (Tabla 4), sin embargo, en la tercera molar izquierda se observó que no hubieron diferencias entre la edad estimada con el método de ligamento periodontal y la edad cronológica en toda la muestra y en el sexo femenino, mientras que al utilizar el método de la pulpa radicular no se encontraron diferencias en el sexo masculino (Tabla 5).

La correlación entre la edad estimada el estudio radiográfico del ligamento periodontal de la pieza 38 en el sexo femenino es positiva moderada y en el sexo masculino es positiva alta y en la 48 en el sexo masculino y femenino es positiva alta, en referencia a la correlación entre la edad estimada por el estudio radiográfico de la pulpa radicular de la pieza 38 y 48 según sexo masculino y femenino es positiva alta (Tabla 6).

\section{Discusión}

El examen del tercer molar puede establecer una precisión razonable de la probabilidad de que una persona tenga al menos 18 años de edad ${ }^{14}$, teniendo en cuenta que la estimación de la edad es importante por razones jurídicas al ser aplicado en la investigación de procedimientos criminales en el derecho penal ${ }^{11}$. Una limitación en el establecimiento de la edad es el empleo de

Tabla 3. Correlación entre la edad cronológica, el estadio del ligamento periodontal y de la pulpa radicular

\begin{tabular}{lccccc}
\hline & Edad cronológica & \multicolumn{2}{c}{ Estudio del ligamento periodontal } & \multicolumn{2}{c}{ Estudio de la pulpa radicular } \\
\hline Sexo & Diente & rho $^{*}$ & $\mathbf{p}$ & rho* $^{*}$ & $\mathbf{p}$ \\
\hline \multirow{2}{*}{ Masculino } & 38 & 0,570 & 0,001 & 0,510 & 0,001 \\
& 48 & 0,601 & 0,001 & 0,601 & 0,001 \\
\multirow{2}{*}{ Femenino } & 38 & 0,466 & 0,001 & 0,629 & 0,001 \\
& 48 & 0,494 & 0,001 & 0,515 & 0,001 \\
\hline
\end{tabular}

*Correlación de Spearman

Tabla 4. Diferencia entre la edad cronológica y estimada por ambos métodos en la tercera molar inferior derecha

\begin{tabular}{|c|c|c|c|c|c|c|c|c|}
\hline \multirow[t]{2}{*}{ Sexo } & \multicolumn{2}{|c|}{ Edad cronológica } & \multicolumn{3}{|c|}{ Estudio del ligamento periodontal } & \multicolumn{3}{|c|}{ Estudio de la pulpa radicular } \\
\hline & Media & Mediana & Media & Mediana & $\mathrm{p}^{*}$ & Media & Mediana & $\mathrm{p}^{+}$ \\
\hline En general & 30,73 & 26,60 & 28,11 & 23,50 & $<0,001$ & 30,31 & 32,30 & 0,561 \\
\hline Masculino & 31,31 & 27,88 & 28,40 & 30,90 & 0,001 & 30,21 & 32,30 & 0,395 \\
\hline Femenino & 30,06 & 26,41 & 27,76 & 23,50 & 0,010 & 30,43 & 33,30 & 0,055 \\
\hline
\end{tabular}

*Edad cronológica-edad estimada por estudio del ligamento periodontal

+Edad cronológica-edad estimada por estudio de la pulpa radicular

Tabla 5. Diferencia entre la edad cronológica y estimada por ambos métodos en la tercera molar inferior izquierda

\begin{tabular}{|c|c|c|c|c|c|c|c|c|}
\hline \multirow[t]{2}{*}{ Sexo } & \multicolumn{2}{|c|}{ Edad cronológica } & \multicolumn{3}{|c|}{$\begin{array}{l}\text { Estudio del ligamento periodontal } \\
\text { Edad estimada }\end{array}$} & \multicolumn{3}{|c|}{$\begin{array}{l}\text { Estudio de la pulpa radicular } \\
\text { Edad estimada }\end{array}$} \\
\hline & Media & Mediana & Media & Mediana & $\mathrm{p}^{*}$ & Media & Mediana & $\mathrm{p}^{+}$ \\
\hline En general & 30,73 & 26,60 & 28,95 & 30,30 & 0,170 & 31,18 & 31,80 & 0,007 \\
\hline Masculino & 31,31 & 27,88 & 29,26 & 30,30 & 0,039 & 31,12 & 32,70 & 0,384 \\
\hline Femenino & 30,06 & 26,41 & 28,60 & 24,10 & 0,209 & 31,25 & 31,80 & 0,002 \\
\hline
\end{tabular}

*Edad cronológica-edad estimada por estudio del ligamento periodontal

+Edad cronológica-edad estimada por estudio de la pulpa radicular

Tabla 6. Correlación entre la edad cronológica, el estadio del ligamento periodontal y de la pulpa radicular

\begin{tabular}{|c|c|c|c|c|c|}
\hline \multicolumn{2}{|c|}{ Edad cronológica } & \multicolumn{2}{|c|}{ Estudio del ligamento periodontal } & \multicolumn{2}{|c|}{ Estudio de la pulpa radicular } \\
\hline Sexo & Diente & $\mathbf{r}^{*}$ & $\mathrm{p}$ & $\mathbf{r}^{*}$ & $\mathrm{p}$ \\
\hline \multirow{2}{*}{ Masculino } & 38 & 0,648 & 0,001 & 0,679 & 0,001 \\
\hline & 48 & 0,651 & 0,001 & 0,640 & 0,001 \\
\hline \multirow{2}{*}{ Femenino } & 38 & 0,582 & 0,001 & 0,627 & 0,001 \\
\hline & 48 & 0,626 & 0,001 & 0,607 & 0,001 \\
\hline
\end{tabular}

*Correlación de Pearson 
metodologías de grupos poblacionales heterogéneos, la cual está influenciado fundamentalmente por la variabilidad genética y los factores ambientales ${ }^{14}$.

Los resultados obtenidos a través de la correlación y diferenciación entre la edad cronológica y la estimación de la edad según el estudio radiográfico establecen que en la tercera molar inferior derecha la pulpa radicular es más precisa que la del ligamento periodontal, mientras que en la tercera molar izquierda el método radiográfico del ligamento periodontal es más precisa que el de la pulpa radicular. Probablemente los resultados son consecuencia de la preponderancia indistinta de uno de los lados mandibulares por presentar cambios biológicos durante el proceso de envejecimiento, por la alteración en la capacidad reparadora del ligamento periodontal siendo menos crítica con la edad a diferencia de la pulpa dental ${ }^{15}$, por estar asociado a una alteración del espesor del ligamento periodontal en algún momento determinado por las funciones masticatorias del individuo, por inflamación crónica ${ }^{16}$, o por alguna alteración en la pulpa radicular que es un tejido menos metabólico que la pulpa coronal formando mayor dentina secundaria ${ }^{17}$.

En relación con el sexo, en el masculino se observó que es más preciso el método del estudio radiográfico de la pulpa radicular en las terceras molares inferiores en relación con el ligamento periodontal, a diferencia del sexo femenino, donde el método de la pulpa radicular de la tercera molar inferior derecha es más precisa, mientras que el método del ligamento periodontal es en la tercera molar inferior izquierda. Esta diferencia podría atribuirse a la mayor prevalencia de atrición en dientes posteriores en el sexo femenino ${ }^{18}$, con una posible alteración en la producción de dentina secundaria de la pulpa dentaria.

En la investigación desarrollada en el estudio radiográfico del ligamento periodontal, los valores mínimos de edad en el sexo masculino y en el femenino, establecen menores valores con relación a lo establecido por Timme ${ }^{11}$, posiblemente las diferencias se deban a la etnicidad puesto que examinó radiografías panorámicas de sujetos alemanes, de etnicidad caucásica, mientras que el estudio se desarrolló en una población mestiza. El impacto de la etnicidad en los métodos de evaluación radiográfica que siguen los estándares de rayos X para la estimación de la edad, fue considerado por Pérez-Mongiovi 19 en el 2015, quien examinó radiografías panorámicas de sujetos portugueses. Así mismo, Garamendi y colaboradores ${ }^{20}$, indican que en la estimación de edad pueden ocurrir dos tipos de errores, el técnico y de etnicidad, siendo el error técnico consecuencia de la diferencia de análisis realizado en imágenes radiográficas panorámicas digitales y en radiografías panorámicas convencionales. En este estudio los valores promedio de las edades según el sexo difieren a lo obtenido por Suárez ${ }^{21}$, posiblemente por las diferencias del margen de edad de la muestra de este estudio la cual estuvo entre los 16 a 74 años y para el estudio de Suárez ${ }^{21}$ entre 18 a 44 años, ya que la imagen radiográfica del ligamento periodontal y la pulpa radicular desaparecen algunas veces después de los 20 años, por lo que no se pueden observar en las radiografías debido a un efecto óptico causado por la estrechez de la membrana del ligamento periodontal, además, de la estrechez del lumen de la pulpa radicular al formarse dentina hasta el punto de que esto no es visible.

La investigación confirma que mientras aumenta el estadio del estudio radiográfico del ligamento periodontal y pulpa radicular aumenta también la edad cronológica tanto en el lado derecho como del izquierdo, así como en los estudios de Olze. ${ }^{10-12}$

Sequeira ${ }^{22}$, determina que hay mayor correlación positiva entre el estudio radiográfico del ligamento periodontal y la edad, siendo mayor en el sexo masculino que en el femenino; y Pérez-Mongiovi ${ }^{19}$, determina que hay igual correlación entre el estudio radiográfico de la pulpa radicular y la edad, en el sexo masculino y femenino; al igual que lo obtenido en el presente estudio.

Los resultados evidenciaron una diferencia significativa entre los lados derecho e izquierdo de la tercera molar inferior tanto en el estudio radiográfico del ligamento periodontal como en la pulpa radicular, diferente al hallazgo de Timme ${ }^{11}$ que indica que no hay una diferencia significativa entre los lados en ambos estudios, posiblemente esto se pueda atribuir a la diferencia de protocolos de visualización de radiografías panorámicas con imágenes convencionales e imágenes digitales, teniendo en cuenta que la visualización digital hace uso de algún software que mejora la precisión de los resultados.

En el estudio radiográfico de la pulpa radicular, según los valores mínimos de edad establecidos en el sexo masculino y en el femenino muestran ser menores que Timme ${ }^{11}$, pudiéndose atribuir a la etnicidad, o porque analizó radiografías panorámicas convencionales.

Los valores promedio de las edades según sexo difieren a lo obtenido por Suárez ${ }^{21}$ posiblemente por las diferencias del margen de edad y menor cantidad de la muestra.

En este estudio se evaluó imágenes radiográficas panorámicas digitales, mientras que Lucas ${ }^{23}$, analizó el ligamento periodontal y pulpa radicular en vistas del tomógrafo panorámico dental, a diferencia de otros autores ${ }^{10-12,19}$, quienes analizaron las radiografías panorámicas convencionales. La razón por la que analizan panorámicas es por el ahorro de tiempo, dinero y la no necesidad de exponer al individuo a más radiación siendo más viable la observación de panorámicas que se toman en la mayoría de los casos por motivos de consulta odontológica.

El estadio H de Demirjian fue considerado en este estudio para denominar mejor el criterio de terceras molares inferiores con mineralización completa y ápices cerrados, estableciéndose que la edad cronológica mínima del individuo a quien le pertenece una imagen radiográfica digital de terceras molares inferiores con ápices cerrados fue de 16 años, al igual que en el estudio de Lucas ${ }^{15}$, mientras que en las investigaciones de Timme ${ }^{11}$ y Olze 10-12 los individuos tuvieron la edad mínima de 15 años.

Los resultados podrían ser generalizados a una población con características similares a la del presente estudio. 
Se concluye que el método de estudio radiográfico de la pulpa radicular en la pieza 48 es más eficaz que el ligamento periodontal y en la pieza 38 el estudio radiográfico del ligamento periodontal es más eficaz que el de la pulpa radicular.

\section{Referencias bibliográficas}

1. Zhang J, An Y, Gao LN, Zhang YJ, Jin Y, Chen FM. The effect of aging on the pluripotential capacity and regenerative potential of human periodontal ligament stem cells. Biomaterials. 2012;33(29):6974-86. DOI: 10.1016/j.biomaterials.2012.06.032.

2. Engebretsen L, Steffen K, Bahr R, Broderick C, Dvorak J, Janarv PM, Johnson A, Leglise M, et al. The International Olympic Committee consensus statement on age determination in high-level young athletes. $\mathrm{Br}$ J Sports Med. 2010;44(7):476-84. DOI: 10.1136/ bjsm.2010.073122.

3. Cameriere R, Ferrante L, De Angelis D, Scarpino F, Galli F. The comparison between measurement of open apices of third molars and Demirjian stages to test chronological age of over 18 year olds in living subjects. Int J Legal Med. 2008;122(6):493-7. DOI: 10.1007/s00414-0080279-6.

4. Gustafson G. Age determination on teeth. J Am Dent Assoc. $\quad 1950 ; 41(1): 45-54 . \quad$ DOI:10.14219/jada.archive. 1950.0132 .

5. Knell B, Ruhstaller P, Prieels F, Schmeling A. Dental age diagnostics by means of radiographical evaluation of the growth stages of lower wisdom teeth. Int J Legal Med. 2009;123(6):465-9. DOI: 10.1007/s00414-009-03302.

6. Caldas IM, Júlio P, Simóes RJ, Matos E, Afonso A, Magalhães T. Chronological age estimation based on third molar development in a Portuguese population. Int J Legal Med. 2011;125(2):235-43. DOI: 10.1007/ s00414-010-0531-8.

7. Liversidge HM, Lyons F, Hector MP. The accuracy of three methods of age estimation using radiographic measurements of developing teeth. Forensic Sci Int. 2003 Jan 9;131(1):22-9. DOI:10.1016/s0379-0738(02)00373-0.

8. Bosmans N, Ann P, Aly M, Willems G. The application of Kvaal's dental age calculations technique on panoramic dental radiographs. Forensic Sci Int. 2005;153:208 212. DOI:10.1016/j.forsciint.2004.08.017.

9. Cameriere R, Ferrante L, Belcastro G, Bonfiglioli B, Rastelli E, Cingolani M. Age estimation by pulp/ tooth ratio in canines by periapical X-rays. J Forensic Sci. 2007;52:166-170. DOI: 10.1111/j.15564029.2006.00336.x.

10. Olze A, Solheim T, Schulz R, Kupfer M, Pfeiffer H, Schmeling A. Assessment of the radiographic visibility of the periodontal ligament in the lower third molars for the purpose of forensic age estimation in living individuals. Int J Legal Med. 2010;124(5):445-8. DOI: $10.1007 /$ s00414-010-0488-7.

11. Timme M, Timme WH, Olze A, Ottow C, Ribbecke S, Pfeiffer $\mathrm{H}$, et al. The chronology of the radiographic visibility of the periodontal ligament and the root pulp in the lower third molars. Sci Justice. 2017;57(4):257-61. DOI: 10.1016/j.scijus.2017.03.004.

12. Olze A, Solheim T, Schulz R, Kupfer M, Scmeling A. Evaluation of the radiographic visibility of the root pulp in the lower third molars for the purpose of forensic age estimation in living individuals. Int J Legal Med. 2010;124(3):183-6. DOI: 10.1007/s00414-010-04887.

13. Gómez E. Eficacia del método de Demirjian y Gleiser - hunt modificado en la estimación de la edad a través del estudio de las terceras molares [tesis de título profesional]. [Lima (Perú)]: Facultad de Odontología de la Universidad Nacional Mayor de San Marcos; 2014.

14. Alshihri AM, Kruger E, Tennant M. Western Saudi adolescent age estimation utilising third molar development. Eur J Dent. 2014 Jul;8(3):296-301. DOI: 10.4103/1305-7456.137628.

15. Correa A. La odontología como ciencia forense su relación con la norma ética y la norma jurídica. México: Universidad Autónoma de México; 2002.

16. Morales F. Temas prácticos en Geriatría y Gerontología. Tomo III. San José-Costa Rica: Universidad Estatal a Distancia; 2007.

17. Demirjian A, Goldstein H, Tanner JM. A new system of dental age assessment. Hum Biol. 1973 May;45(2):21127.

18. Cardentey J, Carmona J, González X, Rodríguez R, Labrador D. Atrición dentaria en la oclusión permanente. Rev Ciencias Médicas. 2014 Jul-Aug;18(4):566-573.

19. Pérez-Mongiovi D, Teixeira A, Caldas IM. The radiographic visibility of the root pulp of the third molar as an age marker. Forensic Sci Med Pathol. 2015 Jun;11(3):339-44. DOI: 10.1007/s12024-015-9688-2.

20. Garamendi P, Landa M, Ballesteros J, Solano M. Estimación forense de la edad en torno a los 18 años. Estudio en una población de inmigrantes indocumentados de origen marroquí. Cuad Med Forense. 2003;31:25-35.

21. Suárez D. Reconstrucción osteobiográfica en odontología forense, según el estudio del macizo craneofacial de sujetos adultos [tesis de doctorado]. Lima (Perú): Facultad de Odontología de la Universidad Nacional Mayor de San Marcos; 2013. 135 p.

22. Sequeira CD, Teixeira A, Caldas IM, Afonso A, Perez-Mongiovi D. Age estimation using the radiographic visibility of the periodontal ligament in lower third molars in a Portuguese population. J Clin Exp Dent. 2014 Dec;6(5):546-50.

23. Lucas V, McDonald F, Andiappan M, Roberts G. Dental age estimation: periodontal ligament visibility (PLV)-pattern recognition of a conclusive mandibular maturity marker related to the lower left third molar at the 18-year threshold. Int J Legal Med. 2016 Nov;131(3):797-801. DOI: $10.1007 / s 00414-016-1468-3$. 
